\title{
The property of rayleigh scattering for an anisotropic dielectric ellipsoid
}

\author{
Ying-le $\mathrm{Li}^{1 *}$, Jin $\mathrm{Li}^{2}$, Ming-jun Wang ${ }^{1}$, Qun-feng Dong ${ }^{1}$
}

${ }^{1}$ Institute of Radio Wave Propagation \& Scattering, Xianyang Normal University, China; ${ }^{*}$ Corresponding author: liyinglexidian@yahoo.com.cn

${ }^{2}$ School of Science, Xidian University, Xian, China

Received 13 October 2010; revised 16 November 2010; accepted 20 November 2010.

\begin{abstract}
The distribution of electric field of an anisotropic dielectric elliptic sphere is presented by reforming both the electromagnetic parameter and shape parameter of this ellipsoid based on the scales transformation of electromagnetic theory. The obtained results are tested. The RCS of the anisotropic target is developed. Simulations show that the direction of incident electric field and the dielectric constant tensor influences RCS remarkably but the target' size doesn't have a great effect on it. The obtained results have some significance in the research of electromagnetic scattering by a complicated anisotropic target.
\end{abstract}

Keywords: Anisotropic Dielectric Elliptic Sphere; Scales Transformation; Scattering

\section{INTRODUCTION}

The solutions of the scattering fields from an isotropic dielectric sphere and an isotropic dielectric ellipsoid can be derived with famous Mie theory and approximate method [1-2] respectively as the incident wave propagating in $\mathrm{z}$-direction and polarizing along $\mathrm{x}$-direction. The scattering properties of prolate elliptic sphere and the coated body with plasma are researched with the numerical methods [3-5]. By solving the supper equation and series summation, Flammer [6] has further determined the scattering field and the inner field for the isotropic ellipsoid. The scattering features and relative researching methods for an absorptive ellipsoid are developed [7-8]. The Rayleigh-Gans and scales theory of electromagnetic field can be used for investigating the special shapes such as needle and disc $[9,10]$. In a word, the subjects of electromagnetic scattering and their applications for an isotropic dielectric ellipsoidal sphere are researched in detail. However, those topics of scat- tering features about an anisotropic dielectric elliptic sphere are not solved at length in theory. There are two knots for the situation. The first is that we must know the analytical expression of electromagnetic field inside this target when it is illumined by a wave from arbitrary direction. The second is that the introduced assistant potentials and their wave equations derived in isotropic dielectric are invalid in anisotropic space. It is well known [2] that if the inside field of an anisotropic target was given, so this target's scattering field is determined entirely. In this paper, based on the scales transformation theory of electromagnetic field, the expression of electric field inside an anisotropic dielectric spherical target is presented in detail. The scattering field from an anisotropic target is developed analytically as the incident wave propagating in arbitrary direction. The validity of the obtained results is tested. Finally the influences induced by the dielectric constant tensor and the parameter of shape are simulated respectively. The method used in this article has the characteristic of briefness in computation and distinctness in physical significance.

\section{INVESTIGATION OF ELECTRIC FIELD INSIDE AN ANISOTROPIC DIELECTRIC ELLIPSOID}

\subsection{Reconstruction of an Anisotropic Elliptic Sphere}

There is an anisotropic dielectric elliptic sphere with semi-axes of $a, b$ and $c$ respectively. The center of the sphere and the original of the primary coordinate system $\Sigma$ are located at the same point. The dielectric constant tensor is given as

$$
\varepsilon=\varepsilon_{0}\left[\begin{array}{ccc}
\varepsilon_{1} & 0 & 0 \\
0 & \varepsilon_{2} & 0 \\
0 & 0 & \varepsilon_{3}
\end{array}\right]
$$

The equation of this ellipsoid is given as 


$$
\frac{x^{2}}{a^{2}}+\frac{y^{2}}{b^{2}}+\frac{z^{2}}{c^{2}}=1
$$

where $\varepsilon_{1}, \varepsilon_{2}$ and $\varepsilon_{3}$ are constants without unit. For the electric potential $u(x, y, z)$, we can easy obtain the following equation in the primary coordinate system $\Sigma$

$$
\varepsilon_{1} \varepsilon_{0} \frac{\partial^{2} u}{\partial x^{2}}+\varepsilon_{2} \varepsilon_{0} \frac{\partial^{2} u}{\partial y^{2}}+\varepsilon_{3} \varepsilon_{0} \frac{\partial^{2} u}{\partial z^{2}}=0
$$

We transform expression (2) into the following

$$
\begin{aligned}
& \varepsilon_{a}\left[\frac{\varepsilon_{1} \varepsilon_{0}}{\varepsilon_{a}} \frac{\partial^{2} u}{\partial x^{2}}+\frac{\varepsilon_{2} \varepsilon_{0}}{\varepsilon_{a}} \frac{\partial^{2} u}{\partial y^{2}}+\frac{\varepsilon_{3} \varepsilon_{0}}{\varepsilon_{a}} \frac{\partial^{2} u}{\partial z^{2}}\right]=0, \\
& \varepsilon_{a}=\frac{\varepsilon_{1} \varepsilon_{0}+\varepsilon_{1} \varepsilon_{0}+\varepsilon_{1} \varepsilon_{0}}{3}
\end{aligned}
$$

We introduce a new coordinate system, scale coordinate system $\Sigma$ '. The coordinates of the system are expressed with $x$ ', y' and z' respectively. The relation of coordinates between the two systems is written as

$$
x^{\prime}=\frac{x}{\sqrt{\frac{\varepsilon_{0} \varepsilon_{1}}{\varepsilon_{a}}}}, y^{\prime}=\frac{y}{\sqrt{\frac{\varepsilon_{0} \varepsilon_{2}}{\varepsilon_{a}}}}, z^{\prime}=\frac{z}{\sqrt{\frac{\varepsilon_{0} \varepsilon_{3}}{\varepsilon_{a}}}}
$$

The differential equation of the potential in the scale coordinate system is derived as by putting the above expression into expression (2)

$$
\varepsilon_{a}\left[\frac{\partial^{2} u^{\prime}}{\partial x^{\prime 2}}+\frac{\partial^{2} u^{\prime}}{\partial y^{\prime 2}}+\frac{\partial^{2} u^{\prime}}{\partial z^{\prime 2}}\right]=0
$$

The condition $u=u$ ' is understandable, since the potential is defined as the work done by electric force move a unit charge from one point to the reference point, namely $W / q$, so both the numerator and the denominator are scale invariants $[10,11]$. Expression(3) shows that an anisotropic dielectric ellipsoid in the primary coordinate system is changed into an isotropic dielectric target, the dielectric constant is $\varepsilon_{a}$, in the scale coordinate system. This situation can simplify greatly the electromagnetic scattering problems. Now the elliptic sphere have been reconstructed into the following

$$
\frac{x^{\prime 2}}{a^{\prime 2}}+\frac{y^{\prime 2}}{b^{\prime 2}}+\frac{z^{\prime 2}}{c^{\prime 2}}=1
$$

where the semi-axes are

$$
a^{\prime}=a \sqrt{\frac{\varepsilon_{a}}{\varepsilon_{1} \varepsilon_{0}}}, b^{\prime}=b \sqrt{\frac{\varepsilon_{a}}{\varepsilon_{2} \varepsilon_{0}}}, c^{\prime}=c \sqrt{\frac{\varepsilon_{a}}{\varepsilon_{3} \varepsilon_{0}}}
$$

\subsection{The Expression of Electric Field Inside an Anisotropic Dielectric Ellipsoid}

We suppose that $E_{0}$ is the size of the incident electric filed and $\theta_{0}, \varphi_{0}$ are its direction parameters in the pri- mary coordinate system. So its projections are written as

$$
\begin{aligned}
& E_{0 x}=E_{0} \sin \theta_{0} \cos \varphi_{0}, \\
& E_{0 y}=E_{0} \sin \theta_{0} \sin \varphi_{0}, \\
& E_{0 z}=E_{0} \cos \theta_{0}
\end{aligned}
$$

The standard of length, one of the four measurement benchmarks, is changed in reconstruction of the target, the electric field defined in the primary coordinate system is therefore different with what defined in the scales coordinate system. From the literatures $[10,11]$, the relations between the electric field are obtained as

$$
E_{0 x}^{\prime}=E_{0 x} \sqrt{\frac{\varepsilon_{0} \varepsilon_{1}}{\varepsilon_{a}}}, E_{0 y}^{\prime}=E_{0 y} \sqrt{\frac{\varepsilon_{0} \varepsilon_{2}}{\varepsilon_{a}}}, E_{0 z}^{\prime}=E_{0 z} \sqrt{\frac{\varepsilon_{0} \varepsilon_{3}}{\varepsilon_{a}}}
$$

It is well known that the inside electric field is function of the outside electric field and the dielectric constant $\varepsilon_{r a}=\varepsilon_{a} / \varepsilon_{0}$ for an ellipsoid [1,2]. This field can be written as

$$
E^{\prime}{ }_{x}=\frac{E_{0 x}^{\prime}}{1+L_{x}\left(\varepsilon_{r a}-1\right)}
$$

where

$$
\begin{aligned}
& L_{x}=\frac{a^{\prime} b^{\prime} c^{\prime}}{2} A_{x}^{\prime}, \\
& A_{x}^{\prime}=\int_{0}^{\infty} \frac{d s}{\left(s+a^{\prime 2}\right)\left[\left(s+b^{\prime 2}\right)\left(s+c^{\prime 2}\right)\left(s+a^{\prime 2}\right)\right]^{\frac{1}{2}}}
\end{aligned}
$$

The expressions for $E_{y}^{\prime}$ and $E_{z}^{\prime}$ are easy obtained by exchange $a^{\prime}, b^{\prime}, c^{\prime} . L_{y}, L_{z}$ and $L_{x}$ satisfy $L_{y}+$ $L_{z}+L_{x}=1$. Expression (8) is defined in the scale coordinate system. In the primary coordinate system $\Sigma$, it is changed $[10,11]$ as

$$
E_{x}=\frac{E_{0 x}}{1+L_{x}\left(\varepsilon_{r a}-1\right)}
$$

The other two fields are also obtained

$$
E_{y}=\frac{E_{0 y}}{1+L_{y}\left(\varepsilon_{r a}-1\right)}, E_{z}=\frac{E_{0 z}}{1+L_{z}\left(\varepsilon_{r a}-1\right)}
$$

where

$$
\begin{aligned}
& L_{y}=\frac{a^{\prime} b^{\prime} c^{\prime}}{2} A_{y}^{\prime}, \\
& A_{y}^{\prime}=\int_{0}^{\infty} \frac{d s}{\left(s+b^{\prime 2}\right)\left[\left(s+b^{\prime 2}\right)\left(s+c^{\prime 2}\right)\left(s+a^{\prime 2}\right)\right]^{\frac{1}{2}}} \\
& L_{z}=\frac{a^{\prime} b^{\prime} c^{\prime}}{2} A_{z}^{\prime},
\end{aligned}
$$




$$
A_{z}^{\prime}=\int_{0}^{\infty} \frac{d s}{\left(s+c^{\prime 2}\right)\left[\left(s+b^{\prime 2}\right)\left(s+c^{\prime 2}\right)\left(s+a^{\prime 2}\right)\right]^{\frac{1}{2}}}
$$

When the target is an isotropic one, namely $\varepsilon_{1}=\varepsilon_{2}=\varepsilon_{3}=\varepsilon_{r}$, it is concluded from the definition of $\varepsilon_{a}$ and expression (5) that

$$
\begin{aligned}
& \varepsilon_{r a}=\varepsilon_{r} \\
& a^{\prime}=a, b^{\prime}=b, c^{\prime}=c
\end{aligned}
$$

Now expressions (10) and (11) are the inside electric fields for an isotropic dielectric ellipsoid, which is in agreement with that in the literature.

\section{THE RAYLEIGH SCATTERING FEATURE OF AN ANISOTROPIC DIELECTRIC ELLIPSOID}

The scattering field from an anisotropic dielectric target is derived as following by using the researching method in literature [2]

$$
\mathbf{E}_{\mathrm{s}}=\mathbf{f}(\hat{i}, \hat{r}) \frac{e^{j k r}}{r}
$$

where

$$
\mathbf{f}(\hat{i}, \hat{r})=\frac{k^{2}}{4 \pi} \int_{v}\left\{-\hat{r} \times\left[\hat{r} \times\left(\varepsilon_{\mathrm{r}} \cdot \mathbf{E}-\mathbf{E}\right)\right]\right\} e^{-j k r^{\prime} \cdot \hat{r}} d v^{\prime}
$$

is the amplitude of scattering field and $\mathbf{E}$ is the electric field inside the ellipsoid. By putting expressions (10) (11) into above, we obtain the following expression

$$
\begin{gathered}
\mathbf{f}(\hat{i}, \hat{r})=\frac{k^{2}}{4 \pi} \int_{v}\left\{-\hat{r} \times\left[\hat{r} \times\left(E_{y}\left(\varepsilon_{2}-1\right) \hat{y}+E_{x}\left(\varepsilon_{1}-1\right) \hat{x}\right)\right.\right. \\
\left.\left.\left.+E_{z}\left(\varepsilon_{3}-1\right) \hat{z}\right)\right]\right\} e^{-j k r^{\prime} \cdot \hat{r}} d v^{\prime}
\end{gathered}
$$

For Rayleigh scattering, $k r^{\prime}=\frac{2 \pi}{\lambda} r^{\prime} \ll 1$, the amplitude is further derived as

$$
\begin{aligned}
\mathbf{f}(\hat{i}, \hat{r})=\frac{k^{2} \mathrm{~V}}{4 \pi} & \left\{-\hat{r} \times\left[\hat{r} \times\left(E_{y}\left(\varepsilon_{2}-1\right) \hat{y}+E_{x}\left(\varepsilon_{1}-1\right) \hat{x}\right.\right.\right. \\
& \left.\left.\left.+E_{z}\left(\varepsilon_{3}-1\right) \hat{z}\right)\right]\right\}
\end{aligned}
$$

where $V=(4 \pi / 3) a b c$. Vectors $\hat{r}$ and $\hat{i}$ are the unit vectors respectively in the scattering direction and the incident electric field direction. Letting

$$
\begin{aligned}
\hat{r} & =\sin \theta \cos \phi \hat{x}+\sin \theta \sin \phi \hat{y}+\cos \theta \hat{z} \\
& =r_{x} \hat{x}+r_{y} \hat{y}+r_{z} \hat{z}
\end{aligned}
$$

So the amplitude is written as

$$
\mathbf{f}(\hat{i}, \hat{r})=\frac{k^{2} \mathrm{~V}}{4 \pi}\left[\left(E_{y}\left(\varepsilon_{2}-1\right) \hat{y}+E_{x}\left(\varepsilon_{1}-1\right) \hat{x}+E_{z}\left(\varepsilon_{3}-1\right) \hat{z}\right)\right.
$$

$$
\left.-\hat{r}\left(E_{y}\left(\varepsilon_{2}-1\right) r_{y}+E_{x}\left(\varepsilon_{1}-1\right) r_{x}+E_{z}\left(\varepsilon_{3}-1\right) r_{z}\right)\right]
$$

If the dielectric constant tensor is a real number, the differential scattering cross section is presented as

$$
\begin{aligned}
\sigma_{\mathrm{d}}= & |\mathbf{f}(\hat{i}, \hat{r})|^{2}=\frac{k^{4} V^{2}}{(4 \pi)^{2}} \\
& \times\left[\left|E_{x}\left(1-r_{x}^{2}\right)\left(\varepsilon_{1}-1\right)-r_{x} r_{y} E_{y}\left(\varepsilon_{2}-1\right)-r_{x} r_{z} E_{z}\left(\varepsilon_{3}-1\right)\right|^{2}\right. \\
& +\left|E_{y}\left(1-r_{y}^{2}\right)\left(\varepsilon_{2}-1\right)-r_{x} r_{y} E_{x}\left(\varepsilon_{1}-1\right)-r_{y} r_{z} E_{z}\left(\varepsilon_{3}-1\right)\right|^{2} \\
& \left.+\left|E_{z}\left(1-r_{z}^{2}\right)\left(\varepsilon_{3}-1\right)-r_{x} r_{z} E_{x}\left(\varepsilon_{1}-1\right)-r_{y} r_{z} E_{y}\left(\varepsilon_{2}-1\right)\right|^{2}\right]
\end{aligned}
$$

It can be seen from (16) that there are two parts in the differential scattering cross section, the first part is only relative to the incident direction, the second part is relative to both incident direction and the observing azimuth angle. From expression (16) it follows that when the incident electric field $\mathbf{E}_{0}$ is in the z-direction. Namely $\mathrm{E}_{0 \mathrm{x}}=\mathrm{E}_{0 \mathrm{y}}=0, \mathrm{E}_{0 \mathrm{z}}=\mathrm{E}_{0}, \varepsilon_{1}=\varepsilon_{2}=\varepsilon_{3}=\varepsilon$, the differential scattering cross section is changed into

$$
\sigma_{\mathbf{d}}=|\mathbf{f}(\hat{i}, \hat{r})|^{2}=\frac{k^{4} \mathbf{V}^{2}}{(4 \pi)^{2}}\left|\frac{3 E_{0}(\varepsilon-1)}{2+\varepsilon}\right|^{2} \sin ^{2} \theta
$$

This is in agreement with what in references [2]. By using the relation $\sigma_{\mathrm{s}}=\int_{\Omega} \sigma_{d} d \Omega$ between the scattering cross section and differential scattering cross section, we obtain the RCS as

$$
\sigma=\frac{6 \mathrm{k}^{4} \mathrm{~V}^{2}}{5 \pi}\left[\left|\left(\varepsilon_{1}-1\right) \mathrm{E}_{\mathrm{x}}\right|^{2}+\left|\left(\varepsilon_{2}-1\right) \mathrm{E}_{\mathrm{y}}\right|^{2}+\left|\left(\varepsilon_{3}-1\right) \mathrm{E}_{\mathrm{z}}\right|^{2}\right]
$$

In Figure 1, the operating frequency is $0.03 \mathrm{GHz}$ and the sizes of the ellipsoid are $a=1 \mathrm{~m}, b=1.3 \mathrm{~m}, c=1.5 \mathrm{~m}$, the incident angle $\theta_{0}=\pi / 3, \phi_{0}=\pi / 4$. So the Rayleigh condition is satisfied. It is concluded that The RCS will augment when the dielectric constant is augment. Since the incident angle and outside electric field are given, the electric dipole is proportional to the size of dielectric constant. So the radiation is big and the scattering field is as well as big. The dielectric tensor used in Figure 2 and Figure 3 is the same as used in Figure 1. The axes and the operating frequency in both figures are respectively $c=0.001 \mathrm{~m}, f=1.5 \mathrm{GHz}$ and $c=1 \mathrm{~m} c=1.5 \mathrm{~m}$, $f=0.03 \mathrm{GHz}$. In Figure $2 \mathrm{a} \sim \mathrm{b} \gg \mathrm{c}$, it is thus approximately consider the ellipsoid as an anisotropic disc. In Figure $3 \mathrm{c} \gg$ a, $\mathrm{c} \gg$ b, it is so approximately consider the ellipsoid as an anisotropic thin stick. It is seen 


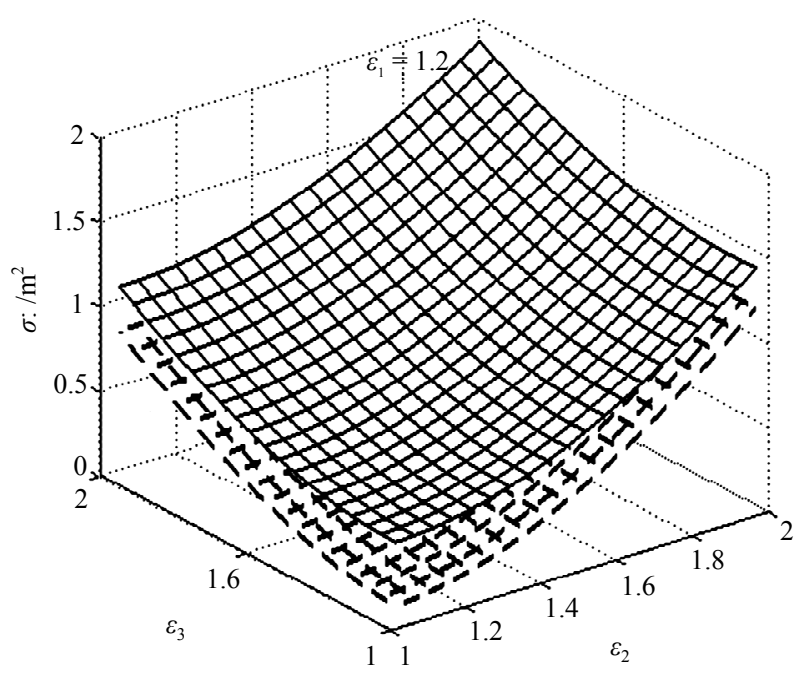

Figure 1. Effect on RCS by the dielectric tensor.

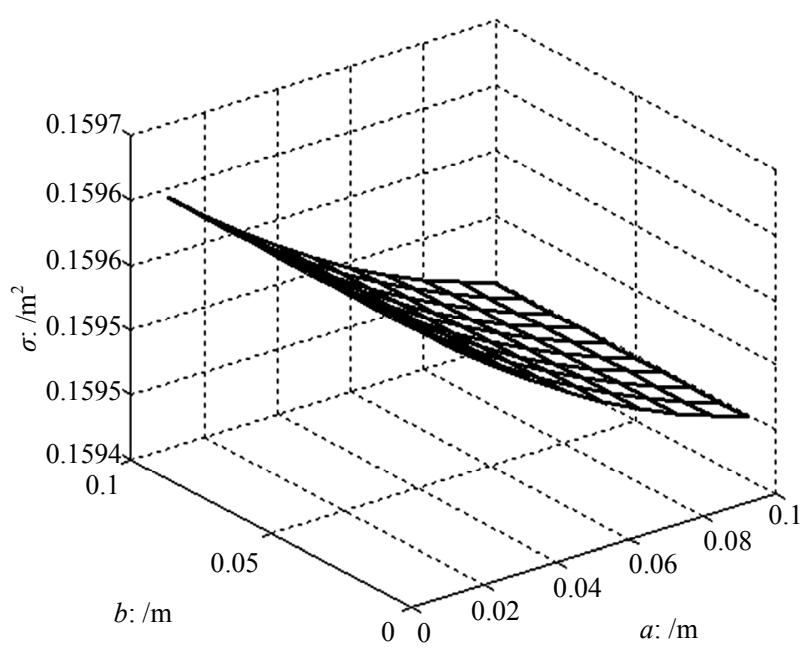

Figure 2. Effect on RCS of a disc by the shape.

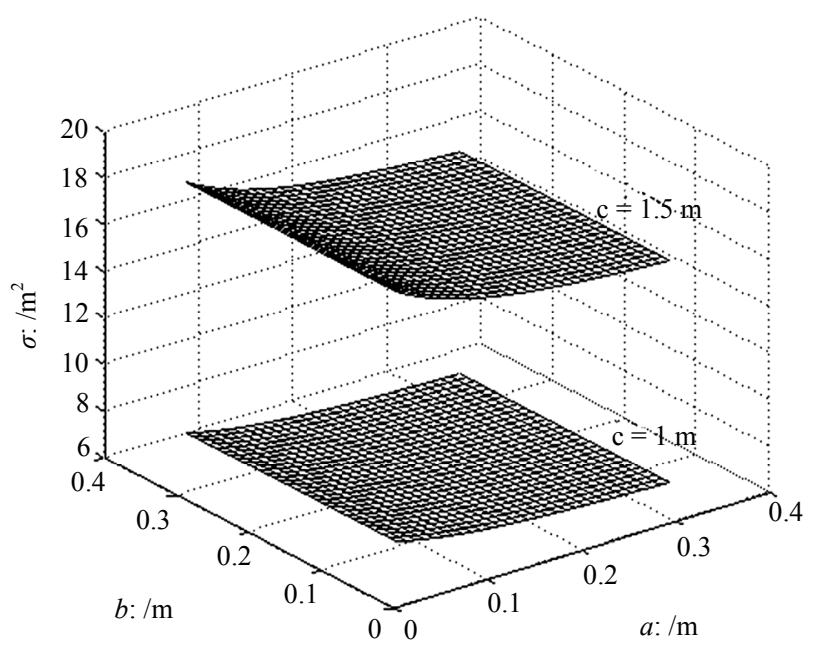

Figure 3. Effect on $\sigma$ of thin stick by shape. that the transverse sizes have not greatly influence the scattering since the transverse dielectric constants are small. In Figure 4 the parameters are used as $a=0.1 \mathrm{~m}$, $b=0.12 \mathrm{~m}, c=0.15 \mathrm{~m}, f=0.3 \mathrm{GHz}$. The dielectric tensor is taken as $\varepsilon_{1}=1.2, \varepsilon_{2}=1.4, \varepsilon_{3}=1.6$. It is shown that the scattering changes greatly with angle $\theta_{0}$, when $\theta_{0}$ is augment, the scattering cross section is decrease. We know that the dielectric constant in z-direction is big, so the projection of electric field in this direction is small as the angle being increase. The electric dipole in the direction is small and the radiation is therefore weakened.

\section{CONCLUSIONS}

In this paper, the expression of electric field of an anisotropic dielectric ellipsoid is presented by reforming both the ellipsoid's electromagnetic parameter and the shape parameter based on the scales transformation of electromagnetic theory. Its correctness is in agreement with literature when the dielectric tensor is an isotropic dielectric. The differential scattering cross section and the RCS are developed in detail. Finally the effects induced by the incident angle, shape and dielectric tensor are computed. Simulations show that the factors such as direction of incident electric field and dielectric tensor have a biggish influence on the scattering features, the shape parameters have not a great effect on them. Many targets such as the dust particles, the pathological organism are generally anisotropic ones, so the obtained results have established a base for researching the Rayleigh scattering property of these kind objects. It is a challenging subject to investigate analytically the scattering field from an anisotropic ellipsoid. When a time varying electromagnetic wave irradiates an anisotropic medium target, how to use the scale transformation theory

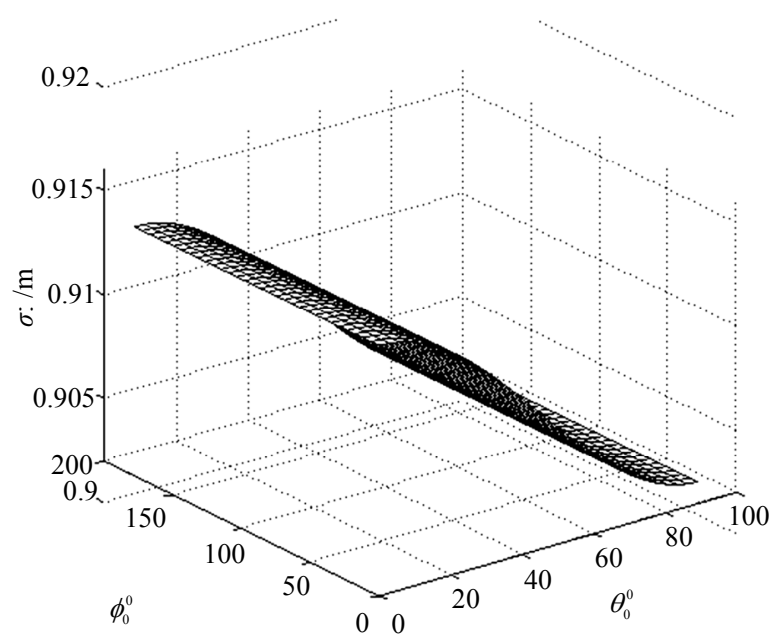

Figure 4. Effect on $\sigma$ by incident angle. 
to study its analytical scattering feature is our next research subject.

\section{ACKNOWLEDGEMENTS}

This project was supported by the National Natural Science Foundation of China (Grant No. 60971079, 60801047), the Natural Science Foundation of Shaanxi Province (Grant No. 2009JM8020) and Natural Science Foundation of Shaanxi Educational Office (Grant No. 09JK800)

\section{REFERENCES}

[1] Sun, X.-M., Shen, J. and Wei, P.-Y. (2009) Light scattering by a spheroid particle with many densely packed inclusions. Acta Physica Sinica, 58, 6222-6226.

[2] Ishimaru, A. (1978) Wave propagation and scattering in random dielectric, Part I. Academic Press, New York.

[3] Shao, S.Y. and Huang, Y.B. (2009) Phase function of prolate spheroidic Mono-Disperse Aerosol particles. Acta Optica Sinica, 29, 100-112.

[4] Gong, L. and Wu, Z.S. (2010) Polarized light scattering from a spheroid particle on or near a wafer. High Power
Laser and Particle Beams, 22, 1393-1396. doi:10.3788/HPLPB20102206.1393

[5] Wang, R., Guo, L.-X. and Yang, G. (2008) Investigation on the electromagnetic scattering from the spheroid covered with the plasma. Journal of Xidian University, 35, 903-909.

[6] Flammer, C. Spheroidal wave function. Stanford University Press, Palo Alto, 1957.

[7] Asano, S. and Yamamoto, G. (1979) Light scattering properties of spheroidal particles. Applied Optics, 18, 712-723. doi:10.1364/AO.18.000712

[8] Asano, S. and Yamamoto, G. (1980) Light scattering by randomly oriented spheroidal particles. Applied Optics, 19, 962-974. doi:10.1364/AO.19.000962

[9] Kerker, M. (1969) The scattering of light and other electromagnetic radiation. Academic Press, New York.

[10] Li, Y.L. and Huang, J.Y. (2005) The scale- transformation of electromagnetic theory and its applications. Chinese Physics, 14.

[11] Li, Y.L. and Huang, J.Y. (2006) Scales transformation theory of electromagnetic field and its application. Xidian University. 